\title{
ESTUDIO COMPARATIVO DE LA CALIDAD DE VIDA DE NIÑOS Y ADOLESCENTES ESCOLARIZADOS DE LIMA Y ANCASH
}

\section{Comparative study of the quality of life of children and adolescents from schools of Lima and Ancash}

\author{
Noemí Sotelo López , Lidia Sotelo López, \\ Sergio Dominguez Lara, Miguel Barboza P.
}

\begin{abstract}
Resumen
Se toman en consideración los planteamientos de Shalock en función a calidad de vida, considerando indicadores tanto para niños como para adolescentes. Objetivo: determinar si existen diferencias en cuanto a la calidad de vida en niños y adolescentes escolarizados de Lima y las zonas rurales de Áncash. Método: Se evaluó a un total de 347 adolescentes y 307 niños entre 8 y 18 años de Lima y Ancash, a través del cuestionario Cuestionario de Calidad de Vida Adolescente y Cuestionario de Calidad de Vida Infantil. Resultados: se reportan diferencias significativas entre niños de Lima y Ancash con relación a los indicadores de calidad de vida, y entre adolescentes de Lima y Ancash, en las dimensiones Desarrollo Personal y Bienestar Material. Conclusión: Los indicadores servirán de base para realizar propuestas dirigidas a mejorar las áreas deficitarias, y potenciar las que estén favoreciendo a las personas.
\end{abstract}

Palabras clave: Calidad de vida, niñez, adolescencia

\begin{abstract}
They are considered Shalock approaches according to quality of life, whereas indicators both for children and for adolescents. Objective: determine if there are differences in the quality of life in children and adolescents from schools of Lima and Ancash. Method: It assessed a total of 307 children and 347 adolescents between 8 and 18 years of Lima and Ancash, through the questionnaire of quality of life for adolescents and questionnaire of quality of life for children. Results: are reported significant differences between children of Lima and Ancash in relation to quality of life indicators, and adolescents in Lima and Ancash, in the personal development and well-being Material dimensions. Conclusion: The indicators will form the basis for proposals to improve the deficit areas and to enhance that are helping people.
\end{abstract}

Key words: Quality of life, childhood, adolescence.

* Decana del Colegio de Psicólogos del Perú-CDR-I. Docente de la Facultad de Psicología de la UNMSM y UIGV. E-mail: noesotelo2002@yahoo.es 


\section{INTRODUCCIÓN}

Cuando se habla de calidad de vida, se hace referencia al grado en que las personas tienen experiencias significativas que valoran, las cuales las habilitan para avanzar hacia una vida plena con relaciones significativas en diversos contextos: donde viven, donde trabajan y donde juegan (Verdugo, 2004, p. 5). En tal sentido, se experimenta cuando las necesidades de las personas se satisfacen y cuando uno tiene la oportunidad de buscar un enriquecimiento en las principales áreas de la vida, considerando además la presencia de componentes tanto objetivos como subjetivos (Shalock, 2010, p. 12).

En cuanto a los desarrollos actuales del concepto calidad de vida, la selección de indicadores se apoya en las dimensiones descritas por Shalock a lo largo de sus numerosas publicaciones: Bienestar e inclusión social, bienestar material, desarrollo personal, bienestar emocional, derechos y bienestar físico. La ventajas está en que estos indicadores guían el análisis situaciones y problemas concretos de los niños y niñas en relación a algunos aspectos referidos a la calidad de las relaciones personales en el seno de la vida en el hogar y con los amigos, que Shalock llama de bienestar e inclusión social; la forma en que éstos satisfacen las necesidades sociales en cuanto a protección, servicios de ayuda en casa, calidad de la vivienda, así como los beneficios recibidos de las políticas de atención a la infancia o de bienestar material; educación y recreación que aluden a desarrollo personal; seguridad social y personal relacionada con bienestar físico.

Del mismo modo, los indicadores de calidad de vida en la adolescencia fueron seleccionados en base a una revisión extensa hecha por Gómez (2003), los cuáles están referidos a Relaciones Interpersonales, es decir, las relaciones valiosas con la familia, así como relaciones de amistad, interacciones sociales con conocidos o compañeros de clase; sin dejar de lado la calidad y satisfacción asociada a las relaciones interpersonales. Bienestar Material, que tiene mucho que ver con la alimentación, alojamiento, confort y estatus socioeconómico de la persona, así como sus ingresos y seguridad económica. Cuando se habla de Desarrollo Personal, se hace referencia a las habilidades y capacidades que podrían brindar oportunidades formativas de crecimiento personal.
El Bienestar Emocional está relacionado con la satisfacción, felicidad, sensación de bienestar general, así como la percepción del cumplimiento de metas y aspiraciones personales. Involucra también las creencias relacionadas con la espiritualidad.

Se relaciona a la Integración/Presencia en la Comunidad el acceso a la comunidad, así como la participación en la misma, lo cual implica la aceptación del estatus dentro del grupo social y la integración. El Bienestar físico tiene que ver con la salud, y el estado físico y mental. Por último, la Autodeterminación tiene que ver con las elecciones personales y la toma de decisiones.

Además, la utilización de este concepto en las escuelas, considerando la niñez y adolescencia, supone una serie de beneficios. Como primera consecuencia positiva, permite avanzar en la educación integral de la persona mediante la implementación de programas que hagan operativa la formulación teórica de este concepto. En segundo lugar, es una buena referencia y guía conceptual de muchas transformaciones y cambios que la escuela necesita realizar para atender las necesidades de los alumnos. Además de ello, puede contribuir a la mejora de la planificación educativa, al desarrollo de modelos específicos de evaluación de programas centrados en la persona, y al incremento de la participación de los usuarios o clientes de la educación en todos los procesos y decisiones que les afectan (Verdugo, 2009, p. 27).

En el Perú, los estudios publicados en el campo de la calidad de vida en la infancia y la adolescencia son escasos y más aún aquellos que describen valores que puedan ser utilizados como referencia para su uso clínico o epidemiológico, para así poder utilizar los indicadores o medidas de evaluación. En este sentido, las encuestas son breves, y no contemplan aquellos aspectos que se recogen a partir de un marco teórico sólido.

En otros países se ha estudiado este aspecto, entre los que destaca Chile, donde Urzúa, Cortés, Prieto, Vega \& Tapia, K. (2009), investigaron la calidad de vida en niños y adolescentes escolarizados, en una muestra de 1678 niños y adolescentes de entre 8 y 18 años de establecimientos públicos, subvencionados y 
particulares de la ciudad de Antofagasta a través del cuestionario KIDSCREEN-27 (Robitail, et al, 2007). Hallaron que los alumnos de mayor edad (12 a 18 años) tuvieron en promedio puntajes inferiores en todas las dimensiones en relación los de menor edad (8 a 11 años). Estas diferencias son estadísticamente significativas en la dimensión física, dimensión psicológica, relación con los padres y autonomía en el ambiente escolar.

En nuestro país, el Instituto Especializado de Salud Mental "Honorio Delgado-Hideyo Noguchi" (2004), en su estudio epidemiológico de salud mental en Huaraz realizado en el año 2003, y en Lima Metropolitana en 2002, usaron para la evaluación de la calidad de vida el Índice de Mezzich (Schwart, ZapataVega, Mezzich \& Mazzotti, 2006), el cual se compone de una escala de intervalo de 1 a 10 , ubicándose la muestra de adolescentes de Huaraz estudiada en un nivel aceptable (7.9), y los adolescentes de Lima Metropolitana, en un nivel similar (7.83), según los resultados proporcionados.

En este contexto, el presente trabajo reportará valores que puedan ser utilizados como referencia en la práctica clínica, en estudios poblacionales, en la descripción de la calidad de vida percibida por los niños y adolescentes de este estudio.

La investigación proyectada tiene como objetivo determinar si existen diferencias en cuanto a la calidad de vida en niños y adolescentes escolarizados de Lima y las zonas rurales de Áncash, con respecto a la ciudad de procedencia, considerando como aspecto fundamental la diferencia que existe entre la capital del país, y las zonas alejadas en lo que respecta al acceso a servicios educativos y de salud de calidad, conociendo que la exposición a un bajo nivel educativo, tanto parental como institucional, se asocia a una disminuida calidad de vida en la infancia, mientras que el acceso reducido a recursos materiales (y por consiguiente sociales) pueden conducir a una calidad de vida inferior especialmente en la adolescencia (Ruden \& Gosch, 2006).

\section{MÉTODO}

Lainvestigación es de tipo descriptivo comparativo (Sánchez y Reyes, 2006). El diseño del estudio es No experimental y Transeccional, cuyo propósito es recolectar los datos describiendo y analizando su incidencia e interrelación en un momento dado (Hernández, Fernández \& Baptista, 2003). Se utilizó un muestro intencional.

\section{Sujetos}

La muestra de niños estuvo conformada por 58 varones y 63 mujeres de Lima, y 95 varones y 91 mujeres de Ancash, siendo la edad promedio fue de 9.85 años y la muestra de adolescentes 47 varones y 27 mujeres de Lima, y 151 varones y 122 mujeres de Ancash, con una edad promedio de 14.73 años.

\section{Instrumentos}

Como instrumentos de evaluación para medir la calidad de vida se utilizó el Cuestionario de Calidad de Vida Infantil (CCVI) de Sabeh, E, Verdugo,M., Contini, E. y Prieto, G. (2009) y el Cuestionario de Calidad de Vida Adolescente (CCVA) de Gómez Vela ,M. y Verdugo, M (2009). Se trata de dos escalas tipo Likert de cuatro opciones de respuesta. En el caso del CCVA, las opciones son Totalmente de acuerdo, Acuerdo, Desacuerdo, Totalmente en desacuerdo; en el CCVI, son Casi siempre, Muchas veces, Pocas veces, Casi nunca.

El CCVA evalúa los siguientes aspectos: Bienestar emocional, Integración a la comunidad, Relaciones interpersonales, Desarrollo personal, Bienestar físico, Autodeterminación y Bienestar material; por su parte, el CCVI evalúa el Bienestar emocional, Relaciones interpersonales, Desarrollo personal, Bienestar físico y Bienestar material. Dichos instrumentos fueron adaptados en nuestro medio antes de aplicarlos.

\section{Procedimiento}

Se contactó a los establecimientos educacionales para invitarlos a participar en el estudio, coordinándose posteriormente la fecha y horarios de aplicación. Dentro de las aulas, no hubo personas que se negaran a completar los cuestionarios, los cuales fueron aplicados en los respectivos centros educativos. El tiempo de duración de la aplicación fue entre 30 y 45 minutos. 
Una vez recogidos los instrumentos, los datos consignados fueron ingresados a una base de datos. Posteriormente a ello, la comparación de grupos fue realizada con la t de Student mediante el paquete estadístico SPSS v.18.0 en español.

\section{RESULTADOS}

Existen diferencias significativas en cuanto a la calidad de vida y autoconcepto de los niños y adolescentes escolarizados de Lima Metropolitana y las zonas rurales de Áncash respecto a la ciudad de procedencia.

Tabla $\mathbf{N}^{\circ} 1$

Comparación de las dimensiones del la Calidad de vida entre niños de Lima y las zonas rurales de Áncash

\begin{tabular}{llll}
\hline Dimensión & Grupo & Media & t(305) \\
\hline \multirow{2}{*}{ Bienestar Emocional } & Lima & 42.10 & $7.55^{* *}$ \\
& Ancash & 37.86 & \\
Relaciones Interpersonales & Lima & 54.07 & $8.27 * *$ \\
& Ancash & 48.02 & $5.65^{* *}$ \\
Desarrollo Personal & Lima & 35.35 & \\
\multirow{2}{*}{ Bienestar Físico } & Ancash & 32.40 & $4.43^{* *}$ \\
& Lima & 19.74 & \\
Bienestar Material & Ancash & 18.52 & $8.30^{* *}$ \\
\hline
\end{tabular}

$* * \mathrm{p}<.001$

Tal como se puede observar, existen diferencias en todas las medias de calidad de vida de niños de Lima y Ancash. Todas las diferencias son estadísticamente significativas y son favorables a los niños de Lima.

Esta diferencia es estadísticamente significativa en la dimensión física $(\mathrm{t}(305)=4.43 ; \mathrm{p}<.001)$; en la dimensión material $(\mathrm{t}(305)=8.30 ; \mathrm{p}<.001)$ y en la dimensión emocional $(\mathrm{t}(305)=7.55 ; \mathrm{p}<.001)$, en donde la media es superior en los niños de Lima. Asimismo, las medidas de Relaciones Interpersonales $(\mathrm{t}(305)=8.27 ; \mathrm{p}<.001)$ y Desarrollo Personal $(\mathrm{t}(305)=$ $5.65 ; \mathrm{p}<.001)$, y favorecen a los escolares limeños. 
Tabla $\mathbf{N}^{\circ} 2$

Comparación de las dimensiones del la Calidad de vida entre adolescentes de Lima y las zonas rurales de Áncash

\begin{tabular}{llll}
\hline Dimensión & Grupo & Media & t(345) \\
\hline \multirow{2}{*}{ Bienestar Emocional } & Lima & 22.81 & 1.27 \\
\multirow{2}{*}{ Integración } & Ancash & 22.25 & \\
& Lima & 32.22 & .049 \\
Relaciones Interpersonales & Ancash & 32.18 & .26 \\
\multirow{2}{*}{ Desarrollo Personal } & Lima & 28.54 & \\
\multirow{2}{*}{ Bienestar Físico } & Ancash & 28.40 & $3.76^{* *}$ \\
Autodeterminación & Lima & 23.28 & \\
& Ancash & 21.89 & 1.94 \\
Bienestar Material & Lima & 16.59 & 1.93 \\
& Ancash & 16.05 & \\
& Lima & 20.24 & $7.09^{* *}$ \\
\hline
\end{tabular}

$* * \mathrm{p}<.001$

Tal como se puede observar, sólo existen diferencias en las medias de calidad de vida de los adolescentes de Lima y Ancash en las dimensiones de Desarrollo Personal $(\mathrm{t}(345)=3.76 ; \mathrm{p}<.001)$ y Bienestar Material $(\mathrm{t}(345)=7.09 ; \mathrm{p}<.001)$, las cuales son estadísticamente significativas y son favorables a los adolescentes de Lima.

\section{DISCUSIÓN}

El aporte fundamental de este estudio es evaluar la calidad de vida bajo indicadores personales y ligados a un marco teórico. De este modo, los indicadores aquí presentados pueden servir como una referencia inicial de la evaluación de la calidad de vida en niños y adolescentes mediante ese modelo, el cual puede ser replicado en otras regiones del Perú.
No se han encontrado, de forma oficial al menos, en la literatura nacional estudios comparativos de calida de vida entre personas provenientes de ámbitos urbanos y rurales, y que además el instrumento de medida se halle ligado a un marco teórico sólido, por lo cual no se podría establecer una comparación en cuanto a metodología utilizada ni a hallazgo; considerándose los estudios del Instituto Especializado de Salud Mental "Honorio Delgado-Hideyo Noguchi", realizados en el año 2003 en Huaraz y en el año 2002 en Lima Metropolitana, comparando dichos estudios se puede apreciar una ligera ventaja a favor de los adolescentes de Lima. Debido a las características del instrumento utilizado no es posible hacer un análisis más profundo de tales resultados.

Con relación a los hallazgos del estudio, en lo que concierne al grupo de niños se halla una clara 
diferencia en todas las áreas evaluadas a favor de los niños provenientes de Lima. Esto se corresponde con lo que plantean Ruden \& Gosch (2006), en la medida que mientras la persona tenga la oportunidad de acceder a servicios que puedan elevar su bienestar, como es educación y salud, la calidad de vida mejorará. En este sentido, son conocidas aquellas limitaciones que tienen las personas que viven en el interior del país, y que dicho impedimento disminuye la calidad de vida percibida, lo cual abarca el aspecto emocional, físico, material, entre otros.

En el grupo de los adolescentes, son sólo dos áreas que se hallan diferencias entre ambos grupos a favor que los adolescentes de Lima: Desarrollo personal y Bienestar Material. En relación al Desarrollo personal, los adolescentes se enfrentan a más demandas académicas y a la toma de decisiones sobre su porvenir profesional (Gómez, 2003). Esto será crítico para aquellos estudiantes que perciban su desempeño académico como insuficiente o no ver clara su alternativa profesional futura puede menguar la satisfacción experimentada en relación con esta dimensión, considerando la posible falta de oportunidad y el estrés que esto conlleva. Asimismo, siguiendo a Ruden \& Gosch (2006), el acceso reducido a recursos materiales pueden conducir a una calidad de vida inferior en la adolescencia, y es lo que indican los resultados de este estudio, en la medida que el Bienestar Material se encuentra disminuido en los adolescentes de las zonas rurales de Ancash.

Estos resultados son para ser tenidos en cuenta, puesto que si tanto la niñez como la adolescencia son etapas de suma importancia en el desarrollo personal, el hecho que algunas de las áreas que componen la calidad de vida se hallan en desventaja, merece la atención de las autoridades y de todas aquellas personas involucradas con esos grupo.

Una limitación compartida que se halla siempre presente sigue siendo el déficit de investigaciones en nuestro país en éste tema, pero sobre todo con instrumentos desarrollados y validados acordes a nuestro contexto, ya que en este caso el instrumento aplicado fue desarrollado y evaluado en una población que es distinta a la nuestra, y eso pudo causar algún sesgo en la respuesta.
Por último, a partir de los resultados se puede realizar una planificación y la gestión en salud al incorporar indicadores positivos de calidad de vida de la infancia y la adolescencia, o bien en la práctica clínica cotidiana, para que así lo que se refiera a calidad de vida no tome una dimensión tan amplia, de modo tal que el profesional que interviene o la institución que evalúa, se enfoque en algunos aspectos importantes que necesitan ser potenciados.

\section{REFERENCIAS}

Gómez,M.(2003).Acercamiento conceptualyempírico a la calidad de vida de alumnos de secundaria. En: González, F., Calvo, I. \& Verdugo, M. (coords). Últimos avances en intervención en el ámbito educativo Actas V Congreso Internacional de Educación Salamanca.

Gómez-Vela, M. y Verdugo, M. (2009). Cuestionario de Evaluación de la Calidad de Vida en Adolescentes (CCVA). Madrid:Ciencias de la Educación Preescolar y Especial.

Hernández, R., Fernández, C. y Baptista, P. (2003). Metodología de la Investigación ( $3^{\circ}$ Ed.). México, D.F.: McGraw Hill.

Instituto Especializado de Salud Mental "Honorio Delgado - Hideyo Noguchi”. (2004) Estudio Epidemiológico de Salud Mental en Huaraz, 2003. Informe General. Lima.

Instituto Especializado de Salud Mental "Honorio Delgado - Hideyo Noguchi" (2002) Estudio Epidemiológico de Salud Mental en Lima Metropolitana. Informe General. Lima.

Robitail, S., Ravens-Sieberer, U., Simeoni, M.C., Rajmil, L., Bruil, J., Power, M., Duer, W., Cloetta, B., Czemy, L., Mazur, J., Czimbalmos, A., Tountas, Y., Hagquist, C., Kilroe, J., Auquier, P. \& The KIDSCREEN Group. (2007). Testing the structural and cross-cultural validity of the KIDSCREEN-27 quality of life questionnaire. Quality of Life Research, 16, 1335-1345.

Ruden, U. \& Gosch, A. (2006). Socioeconomic determinants of health related quality of life in 
childhood and adolescent: results from a European study. Journal Epidemiol Community Health, 60, 130-135.

Sabeh, E., Verdugo, M., Contini, E. y Prieto, G. (2009). Cuestionario de Evaluación de la Calidad de Vida en la Infancia (CVI-CVIP). Madrid: Editorial Ciencias de la Educación Preescolar y Especial.

Sánchez, H. \& Reyes, C. (2006). Metodología y diseños en investigación científica. Lima: Editorial Visión Universitaria.

Shalock, R. (2010). Aplicaciones del paradigma de calidad de vida a las personas con discapacidad intelectual y del desarrollo. Aplicación del paradigma de calidad de vida. VII Seminario de Actualización Metodológica en Investigación sobre Discapacidad SAID (pp. 11-18).

Schwartz K, Zapata-Vega M, Mezzich J, Mazzotti G. (2006). Validation study of the multicultural quality of Life (MQLI) in a Peruvian sample. Revista Brasileira de Psiquiatría, 28(1), 24-32.

Urzúa, A, Cortés, E., Prieto, L. Vega, S. y Tapia, K. (2009). Autoreporte de la Calidad de Vida en Niños y Adolescentes Escolarizados. Revista Chilena de Pediatría, 80(3), 238-244.

Verdugo, M. (2004). Calidad de vida y calidad de vida familiar. II Congreso Internacional de Discapacidad Intelectual: Enfoques $y$ Realidad: Un desafío. Recuperado de: http:// www.pasoapaso.com.ve/CMS/images/stories/ Integracion/cdvfverdugo.pdf,.

Verdugo, M. (2009). El cambio educativo desde una perspectiva de calidad de vida. Revista de Educación, 349, 23-43. Recuperado de: http:// www.revistaeducacion.mec.es/re349/re349.pdf,

Fecha de recepción: 26 de octubre 2012

Fecha de aceptación: 3 marzo 2013 